\title{
Gray solitons on the surface of water
}

\author{
A. Chabchoub, ${ }^{1,2, *}$ O. Kimmoun, ${ }^{3}$ H. Branger, ${ }^{3}$ C. Kharif, ${ }^{3}$ N. Hoffmann, ${ }^{2,4}$ M. Onorato,,${ }^{5,6}$ and N. Akhmediev ${ }^{7}$ \\ ${ }^{1}$ Centre for Ocean Engineering Science and Technology, Swinburne University of Technology, Hawthorn, Victoria 3122, Australia \\ ${ }^{2}$ Department of Mechanical Engineering, Imperial College London, London SW7 2AZ, United Kingdom \\ ${ }^{3}$ Aix Marseille Université, CNRS, Centrale Marseille, IRPHE UMR 7342, 13384, Marseille, France \\ ${ }^{4}$ Dynamics Group, Hamburg University of Technology, 21073 Hamburg, Germany \\ ${ }^{5}$ Dipartimento di Fisica, Università degli Studi di Torino, Torino 10125, Italy \\ ${ }^{6}$ Istituto Nazionale di Fisica Nucleare, INFN, Sezione di Torino, 10125 Torino, Italy \\ ${ }^{7}$ Optical Sciences Group, Research School of Physics and Engineering, Institute of Advanced Studies, \\ The Australian National University, Canberra ACT 0200, Australia
}

(Received 16 October 2013; published 10 January 2014)

\begin{abstract}
The dynamics of surface gravity water waves can be described by the self-defocusing nonlinear Schrödinger equation. Recent observations of black solitons on the surface of water confirmed its validity for finite, below critical depth. The black soliton is a limiting case of a family of gray soliton solutions with finite amplitude depressions. Here, we report observations of gray solitons in water waves, thus, complementing our previous observations of black solitons.
\end{abstract}

DOI: 10.1103/PhysRevE.89.011002

PACS number(s): 05.45.Yv, 47.35.Fg, 92.10.Hm, 92.40.Qk

Dark solitons have been studied intensely for the last two decades. They have been observed experimentally in a variety of nonlinear dispersive media including optical setups [1-4], photonic crystal fibers [5], Bose-Einstein condensates [6,7], plasmas [8,9], and water surface [10]. Similar features of nonlinear wave propagation in all these media have been established within the powerful framework of the nonlinear Schrödinger equation (NLS). Integrability of the NLS provides a fascinating set of exact solutions [11-13]. Although the NLS is an approximation, its exact solutions model the experimental data remarkably well [14]. Applicability of the NLS in hydrodynamics has been established back in the 1970s [15,16]. It describes the dynamics of surface gravity waves in finite or infinite depth, depending on the ratio between the water depth and wavelength. In particular, Yuen and Lake observed bright solitons of the focusing NLS $[15,16]$. However, experimental observations of pulsating bright solutions $[17,18]$, as well as dark solitons in water waves [19] have been reported only recently. The black soliton solution of the defocusing NLS is a special case of a more general family of gray solitons. It can be observed on the surface of water of finite, below critical depth. These solutions are stationary in the frame of reference moving with the wave group velocity. The amplitude of monochromatic waves drops to zero for black solitons, while it is depressed to a finite value in the case of gray solitons. In the present work, we present observations of the family of gray solitons.

The NLS for weakly nonlinear water waves can be derived using the method of multiple scales [20,21]:

$$
-i\left(\frac{\partial A}{\partial t}+c_{g} \frac{\partial A}{\partial x}\right)+\alpha \frac{\partial^{2} A}{\partial x^{2}}+\beta|A|^{2} A=0,
$$

*achabchoub@swin.edu.au where $\alpha=-\frac{1}{2} \frac{\partial^{2} \omega}{\partial k^{2}}$ is the dispersion coefficient, while

$$
\begin{aligned}
\beta= & \frac{\omega k^{2}}{16 \sinh ^{4}(k h)}\left[\cosh (4 k h)+8-2 \tanh ^{2}(k h)\right] \\
& -\frac{\omega}{2 \sinh ^{2}(2 k h)} \frac{\left[2 \omega \cosh ^{2}(k h)+k c_{g}\right]^{2}}{g h-c_{g}^{2}}
\end{aligned}
$$

is the nonlinear coefficient which is a function of $\omega, k$, the group velocity $c_{g}=\frac{\partial \omega}{\partial k}$, and the water depth $h$. Here, $x$ and $t$ are the space and time variables. The frequencies are related to the wave numbers via the dispersion relation $\omega=\sqrt{g k \tanh (k h)}$, where $g$ is the gravitational acceleration. In the limiting case of infinite depth, when $k h \longrightarrow \infty$, the dispersion and nonlinear coefficients can be simplified to $\alpha=\frac{\omega}{8 k^{2}}$ and $\beta=\frac{\omega k^{2}}{2}$, respectively [22]. To first order in steepness, the surface elevation $\eta(x, t)$, which is actually the measurable variable is given by

$$
\eta(x, t)=\operatorname{Re}\{A(x, t) \exp [i(k x-\omega t)]\} .
$$

When $k h>1.363$, the coefficients $\alpha$ and $\beta$ have the same sign and a monochromatic wave train exponentially grows due to the Benjamin-Feir instability [22-24]. Its further evolution can be described using exact breather solutions [25-27]. However, when $k h<1.363$, the nonlinear coefficient $\beta$ changes its sign and regular wave trains are stable to perturbations of any frequency.

The defocusing NLS can be written in the form

$$
i \psi_{T}+\psi_{X X}-2|\psi|^{2} \psi=0 .
$$

It is obtained from (1) by scaling of all three variables [19]. $X$ is now the normalized coordinate in a frame moving with the group velocity and $T$ is the scaled time. We take the amplitude of the carrier $a$ being normalized to one. It can be easily rescaled to an arbitrary value using the scaling transformation [11]. Equation (3) admits a one-parameter family of exact gray soliton solutions:

$$
\begin{aligned}
\psi_{G}= & {[\sin (\vartheta)+i \cos (\vartheta) \tanh \{\cos (\vartheta)[X+2 \sin (\vartheta) T]\}] } \\
& \times \exp (-2 i T+i \chi),
\end{aligned}
$$


(a)
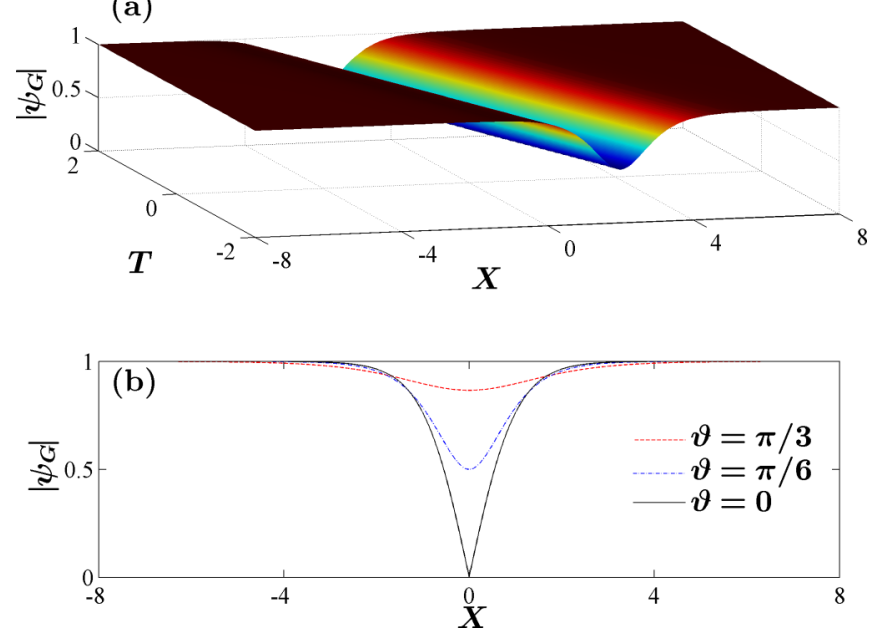

FIG. 1. (Color online) (a) An example of a gray soliton for $a=1$, $\chi=0$, and $\vartheta=\frac{\pi}{6}$. (b) Gray soliton amplitude depressions at $T=0$ for $a=1, \chi=0$, and $\vartheta \in\left\{\frac{\pi}{3}, \frac{\pi}{6}, 0\right\}$.

where $\chi$ is an arbitrary phase shift and $\vartheta$ is the parameter of the family $(-\pi / 2<\vartheta<\pi / 2)$. It controls the minimal amplitude of the soliton $\min |\psi|=|\sin \vartheta|$ and the soliton velocity $v=$ $\sin \vartheta$ which varies from -1 to +1 . The background amplitude of the solution (4) is $a=1$. An example of a gray soliton is shown in Fig. 1. The soliton propagates at a finite angle to the $T$ axis due to nonzero velocity. The direction of propagation depends on the sign of $\vartheta$.

Equation (4) is the simplest form of the gray soliton solution. For $m=\frac{\pi}{2}-\vartheta$ it can also be transformed into

$$
\begin{aligned}
\psi_{G}= & \frac{\exp [-2 i(m)]+\exp \{2 \sin (m)[X+2 \cos (m) T]\}}{1+\exp \{2 \sin (m)[X+2 \cos (m) T]\}} \\
& \times \exp (-2 i T+i \chi),
\end{aligned}
$$

which is more familiar from the works $[25,28]$. Note that the solution given in [25] has a typo.

Gray solitons admit a simple geometric interpretation [29]. Any first order solution of the NLS can be represented by a straight line on the complex plane $(\operatorname{Re} \psi, \operatorname{Im} \psi)$ of the $\psi$ variable [11]. This plane is shown in Fig. 2. The background of the soliton with the unit amplitude is any point on a circle with a unit radius. The argument of this point is the phase of the plane wave at any given fixed $T$. As the plane waves on the left- and on the right-hand sides of the soliton have different phases; they are represented by two different points on the unit circle. Suppose, these are the points $A$ and $B$. Then all complex amplitude points of the solution are located on a straight line $A B$ connecting these two points. It is easy to check that when $T$ is fixed and $X$ is varied, Eqs. (4) and (5) indeed define a straight line between the two points. The arbitrary phase $\chi$ and current time $T$ define the orientation of the straight line on the complex plane. The line rotates when $T$ is evolved but relative positions of all points remain fixed.

From this geometric representation, it is easy to see that the minimal amplitude of the gray soliton is the shortest distance between the origin and the straight line, i.e., it is defined by the line $O C$. This distance is clearly given by $\sin \vartheta$. When the points $A$ and $B$ are on the opposite sides of the circle, the line

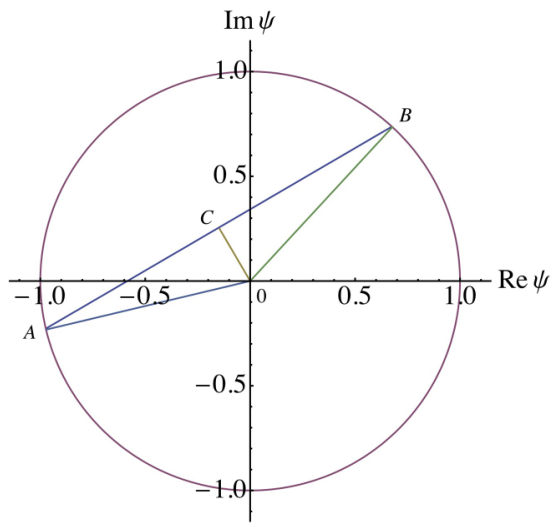

FIG. 2. (Color online) Simple geometric representation of the gray soliton on the complex plane of $\psi$. At any fixed $T$, the solution is represented by the points on the line $A B$. The points $A$ and $B$ represent the plane waves at the left- and at the right-hand sides of the soliton. The point $C$ corresponds to the point of minimal soliton amplitude. Parameter of the family $\vartheta$ is the angle $A$ (or $B$ ). The phase shift between the left- and right-hand sides of the soliton is given by the central angle $(\pi-2 \vartheta)$.

$A B$ passes through the origin and the minimum amplitude is zero. This is the case of the black soliton. This happens when $\vartheta=0$. Then Eq. (4) is transformed into

$$
\psi_{B}=i \tanh (X) \exp (-2 i T+i \chi)
$$

A prominent feature of gray solitons is its related phase shift. This is the angle between the points $A$ and $B$. It is given by $(\pi-2 \vartheta)$. Depending on whether the background plane wave $A$ is on the left- or on the right-hand side, the soliton has positive or negative velocity, respectively. The velocity is zero for the black soliton. Thus, most of the gray soliton parameters can be clearly visualized in this simple geometrical construction. Note also that this description is valid for any amplitude $a$. Parameter $\vartheta$ has the same meaning for any value of $a$.

The experiments have been conducted in the same facility as the one described in [19]. The single computer controlled flap created initial conditions for generating waves. These have been programed using the above formulas at fixed $X$ that correspond to the position of the flap. The wave dynamics has been measured with the resistive wave gauges with sampling frequency $200 \mathrm{~Hz}$. Their positions along the tank are shown in Table I.

In order to generate the gray solitons, we have to satisfy the condition $k h<1.363$. For that purpose, we conducted the experiments for water depth values of $h=0.25 \mathrm{~m}$ and $h=$ $0.20 \mathrm{~m}$ and adapted the wavelengths accordingly. Once a value for the soliton parameter $\vartheta$ is selected, the gray soliton solution $\psi_{G}(X, T)$ is rescaled back to the dimensional units $A_{G}(x, t)$ according to (1). The initial conditions are then determined by

TABLE I. Wave gauge positions along the wave flume.

\begin{tabular}{lcccccccc}
\hline \hline Gauge number & 1 & 2 & 3 & 4 & 5 & 6 & 7 & 8 \\
\hline Position (m) & 1.06 & 4.33 & 7.28 & 7.64 & 7.82 & 8.18 & 10.19 & 12.52 \\
\hline \hline
\end{tabular}




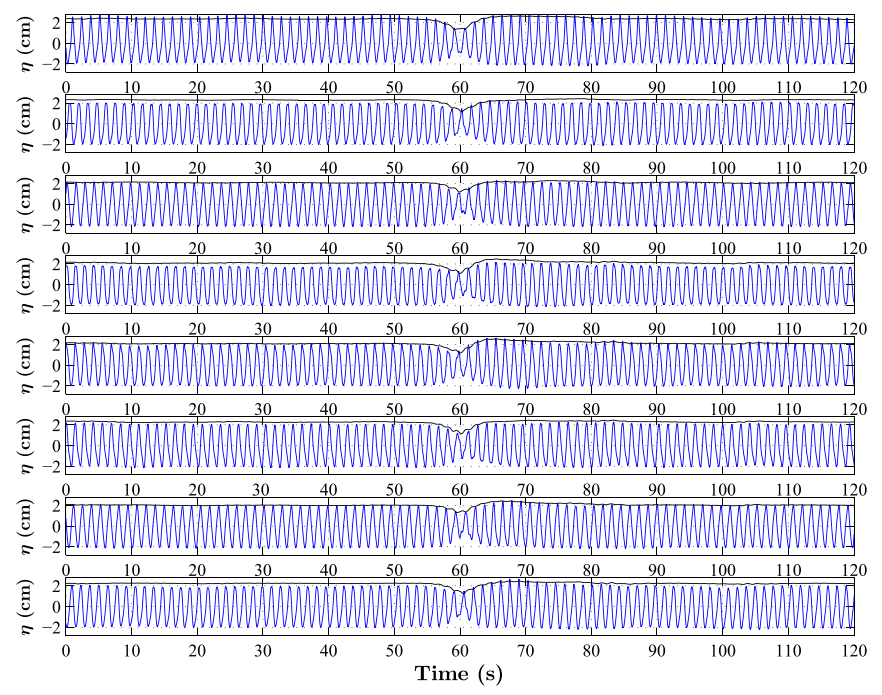

FIG. 3. (Color online) Evolution of a gray soliton along the wave flume with $\vartheta=\frac{\pi}{6}$, for carrier amplitude of $0.02 \mathrm{~m}$, a water depth of $h=0.25 \mathrm{~m}$ and $k h=0.8$. The measurements are shifted in time in order to cancel the combined soliton and the wave group velocity, which are of $1.22 \mathrm{~ms}^{-1}$, and keep the depression of the gray soliton at the central position.

evaluating Eq. (2) at the flap position $x^{*}$. We chose $x^{*}=0$, that is, the signal $\eta_{G}\left(x^{*}=0, t\right)$ applied to the flap before taking into account the wave generator transfer function, is

$$
\eta_{G}\left(x^{*}, t\right)=\operatorname{Re}\left\{A_{G}\left(x^{*}, t\right) \exp \left[i\left(k x^{*}-\omega t\right)\right]\right\} .
$$

We started with a soliton of lowest depression (minimal amplitude) and decreased the depression in the following experiments. Figure 3 shows the evolution of a gray soliton with $\vartheta=\pi / 6$ and the amplitude $0.02 \mathrm{~m}$. The water depth is $h=0.2 \mathrm{~m}$ and $k h=0.8$. For the chosen value of $\pi / 6$ for the parameter $\vartheta$, the depression of the monochromatic wave field

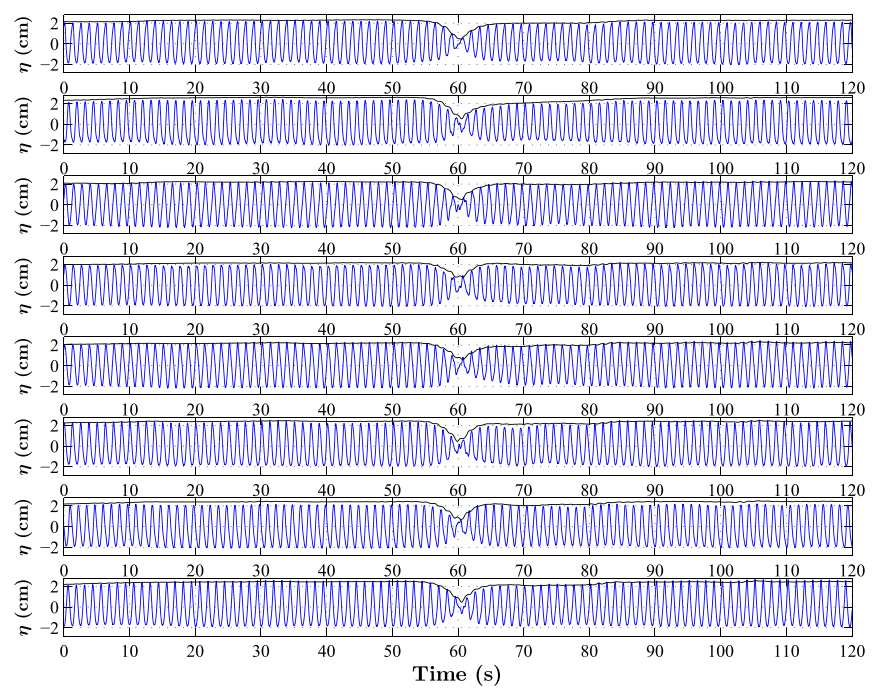

FIG. 4. (Color online) Evolution of a gray soliton with $\vartheta=\frac{\pi}{14}$ and carrier amplitude of $0.02 \mathrm{~m}$, a water depth of $h=0.25 \mathrm{~m}$ and $k h=0.9$. The measurements are shifted in time, as in Fig. 3, with respect to the velocity value of $1.13 \mathrm{~ms}^{-1}$. is expected to be half the amplitude of the carrier wave. As can be seen from Fig. 3, the experiments are in good agreement with this theoretical value. Each upper plot in Fig. 3 is shifted relative to the lower one in order to keep the coordinate with minimum amplitude at the center. The velocity calculated from these shifts agrees well with the theoretical value.

Another example of a gray soliton evolution is shown in Fig. 4. Here, the value of the parameter $\vartheta$ is $\pi / 14$, which corresponds to a depression of almost $80 \%$ below the background amplitude. Thus, the minimal amplitude here is $0.004 \mathrm{~m}$, since the amplitude of the carrier was chosen to be $0.02 \mathrm{~m}$. The water depth in this experiment is $h=0.25 \mathrm{~m}$ and $k h=0.9$. Due to the smallness of the chosen carrier parameters, dictated by the size of the tank, the velocity of the localized depression would not vary significantly compared to the previous case. The experiments confirm the stationary
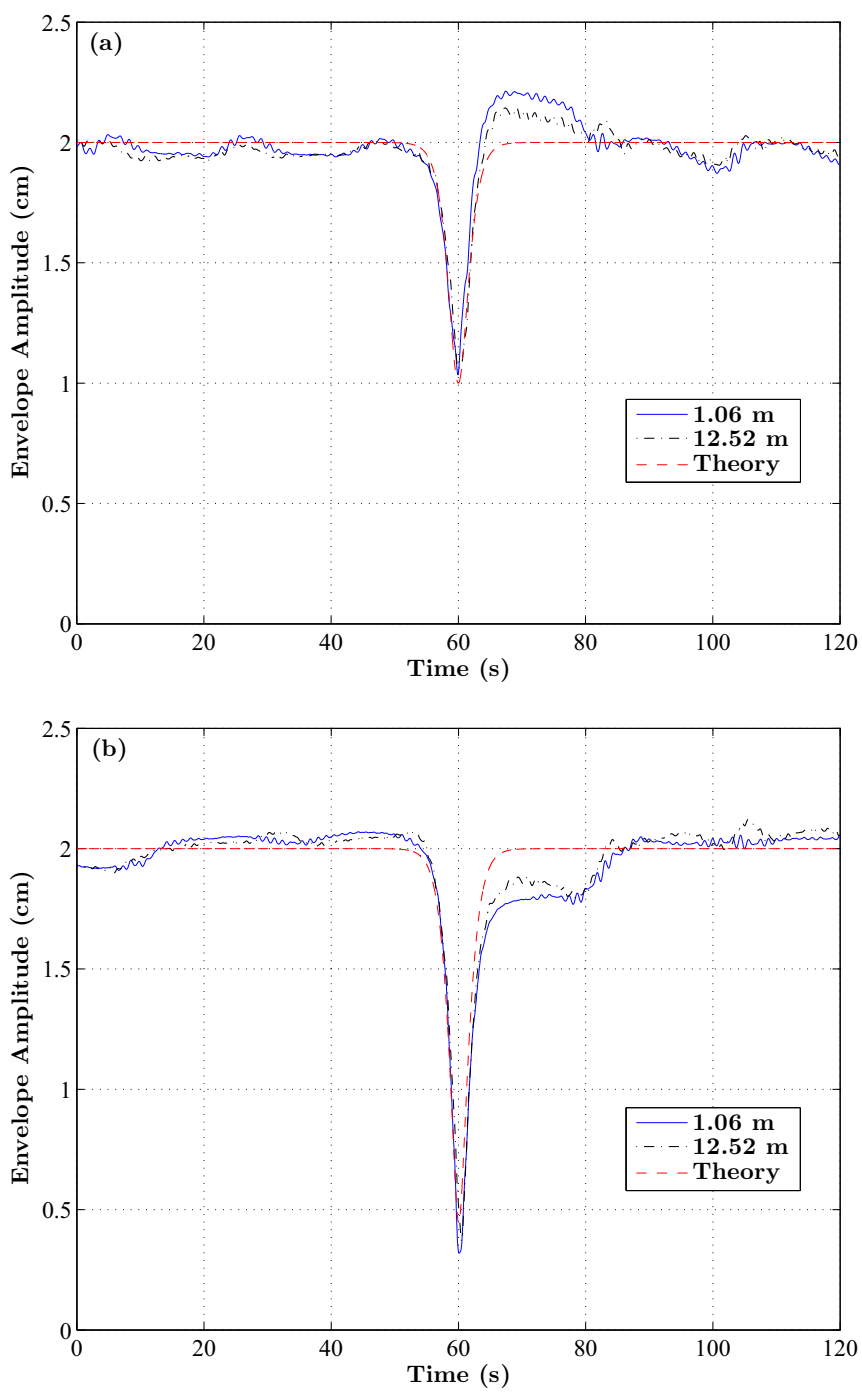

FIG. 5. (Color online) (a) Superposition of the envelopes of the observed gray soliton, shown in Fig. 3, measured by the first wave gauge (solid blue line), and the last wave gauge (black dash-dotted line) with the theoretical envelope (red dashed line). (b) Same as in (a), but for the gray soliton shown in Fig. 4 . 


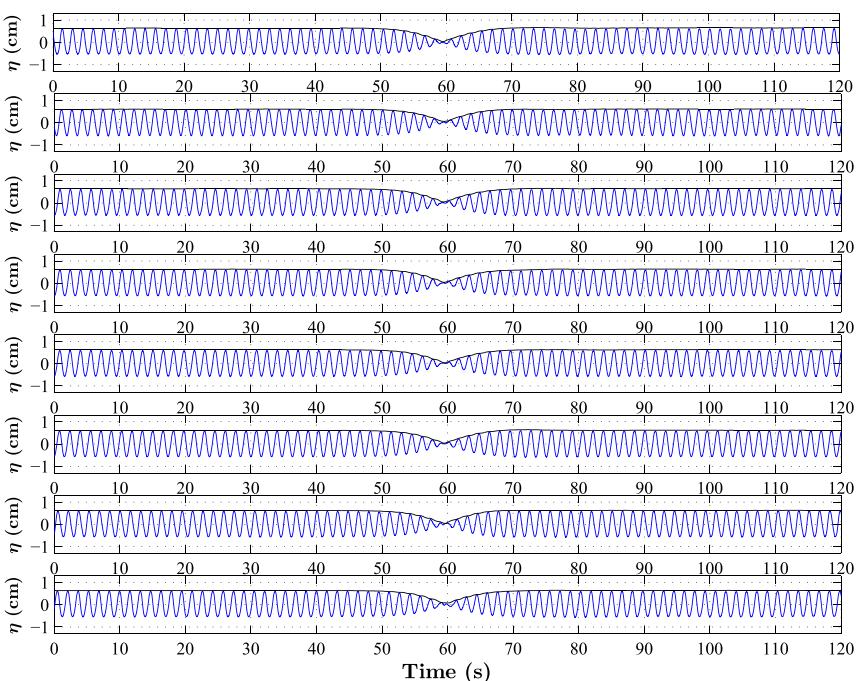

FIG. 6. (Color online) Evolution of a black soliton $(\vartheta=0)$ along the wave flume for carrier amplitude $0.075 \mathrm{~m}$. The water depth is $h=0.2 \mathrm{~m}$ and $k h=0.6$. Here, the group velocity of the background waves, which correspond to $1.18 \mathrm{~ms}^{-1}$, has been canceled to keep the black soliton in the center.

evolution of the gray soliton with the depression remaining nearly constant.

Figures 3 and 4 show clearly that the gray solitons keep their constant profile. In order to better illustrate the steadiness of the soliton, we compare the wave envelopes of the first and last wave gauge measurements in the flume with the theoretical free surface elevation, (2). The results are shown in Fig. 5. Note that the soliton width, i.e., the number of modulated waves, is independent from the choice of the parameter $\vartheta$. As shown in [19], the width of the soliton is only dependent on the steepness of the carrier $a k$, as well as the $k h$ value.

There are unavoidable small variations of the background wave which develop around the soliton. These can be considered as small amplitude perturbations [30]. These perturbations also appear in fiber optics [31]. However, stability of the background relative to small modulations ensures the robustness of the gray soliton located on this background. The phase shift shown in Fig. 2 is a topological structure that cannot be eliminated guaranteeing the stability. The soliton velocity is different from the phase velocity of the carrier. This means that the envelope of the waves is continuously shifting relative to the phase pattern of the carrier waves. Consequently, the point of minimum of the gray soliton continuously moves along the sinusoidal pattern of the carrier wave.

One limitation is the validity of the NLS in terms of $k h$. It has been shown that the NLS cannot be valid for very small $k h$ values [32,33]. In the results shown in Fig. 6, we reached the experimental limit in our observations of dark solitons. Regular wave generation for the single flap wavemaker has been reached for the depth of $h=0.2 \mathrm{~m}$ and $k h=0.6$. Using exact initial conditions we observed generation of black solitons, i.e., $\vartheta=0$, as shown in Fig. 6. Our observations proved the validity of the NLS for $k h$ values as small as 0.6. An exact threshold value could be determined in a more accurate experiment using a piston-type wavemaker [34].

One result of our observations of gray solitons is their asymmetry relative to the soliton center as can be seen from Fig. 5. The deviation from the NLS theory is due to the fact that exact solutions describe the motion of free surface waves (2), while the surface elevation also contains bound waves. Consequently, the experimental amplitudes deviate from those described by the NLS envelope. Black solitons are more sturdy and less sensitive to the presence of the Stokes components as can be seen from Fig. 6. This issue needs more work.

A.C. would like to thank Alexander Babanin, Roger Grimshaw, and Efim Pelinovsky for helpful discussions. A.C. acknowledges partial support from the Australian Research Council (Discovery Projects DP1093349 and DP1093517). N.H. and N.A. acknowledge the support of the Volkswagen Stiftung. N.A. acknowledges support of the ARC (Discovery Project DP110102068) and from the Alexander von Humboldt Foundation. M.O. was supported by ONR Grant No. N000141010991 and by MIUR Grant No. PRIN 2012BFNWZ2. M.O. acknowledges Dr. B. Giulinico for interesting discussions.
[1] P. Emplit et al., Opt. Commun. 62, 374 (1987).

[2] D. Krökel, N. J. Halas, G. Giuliani, and D. Grischkowsky, Phys. Rev. Lett. 60, 29 (1988).

[3] A. M. Weiner et al., Phys. Rev. Lett. 61, 2445 (1988).

[4] E. Smirnov, C. E. Rüter, M. Stepić, D. Kip, and V. Shandarov, Phys. Rev. E 74, 065601(R) (2006).

[5] M. Tlidi and L. Gelens, Opt. Lett. 35, 306 (2010).

[6] L. D. Carr, J. Brand, S. Burger, and A. Sanpera, Phys. Rev. A 63, 051601(R) (2001).

[7] D. J. Frantzeskakis, J. Phys. A: Math. Theor. 43, 213001 (2010).

[8] P. K. Shukla and B. Eliasson, Phys. Rev. Lett. 96, 245001 (2006).

[9] R. Heidemann, S. Zhdanov, R. Sütterlin, H. M. Thomas, and G. E. Morfill, Phys. Rev. Lett. 102, 135002 (2009).

[10] F. Dias and C. Kharif, Ann. Rev. Fluid Mech. 31, 301 (1999).
[11] N. Akhmediev and A. Ankiewicz, Solitons: Non-Linear Pulses and Beams (Champan and Hall, London, 1997).

[12] C. Kharif, E. Pelinovsky, and A. Slunyaev, Rogue Waves in the Ocean (Springer, Heidelberg, NY, 2009).

[13] A. Osborne, Nonlinear Ocean Waves and the Inverse Scattering Transform (Elsevier, Amsterdam, 2010).

[14] A. Slunyaev et al., Phys. Rev. E 88, 012909 (2013).

[15] H. C. Yuen and B. M. Lake, Phys. Fluids 18, 956 (1975).

[16] H. C. Yuen and B. M. Lake, Adv. Appl. Mech. 22, 67 (1982).

[17] A. Chabchoub et al., Phys. Rev. E 86, 056601 (2012).

[18] A. Chabchoub et al., Phys. Rev. Lett. 111, 054104 (2013).

[19] A. Chabchoub et al., Phys. Rev. Lett. 110, 124101 (2013).

[20] H. Hasimoto and H. Ono, J. Phys. Soc. Jpn. 33, 805 (1972).

[21] C. C. Mei, The Applied Dynamics of Ocean Surface Waves, Advanced Series on Ocean Engineering Vol. 1 (World Scientific, 
Singapore, 1989), see: http://www.worldscientific.com/doi/pdf/ 10.1142/9789812796059_fmatter.

[22] V. E. Zakharov, J. Appl. Mech. Tech. Phys. 2, 190 (1968).

[23] M. J. Lighthill, J. Inst. Math. Appl. 1, 269 (1965).

[24] T. B. Benjamin and J. E. Feir, J. Fluid Mech. 27, 417 (1967).

[25] D. H. Peregrine, J. Austral. Math. Soc. Ser. B 25, 16 (1983).

[26] N. Akhmediev, V. M. Eleonskii, and N. E. Kulagin, Sov. Phys. JETP 62, 894 (1985).

[27] N. Akhmediev, V. M. Eleonskii, and N. E. Kulagin, Teor. Mat. Fiz. (USSR) 72, 183 (1987).

[28] V. E. Zakharov and A. B. Shabat, Sov. Phys. JETP 34, 62 (1972).
[29] N. Akhmediev and A. Ankiewicz, Phys. Rev. A 47, 3213 (1993).

[30] M. J. Ablowitz et al., Proc. R. Soc. London, Ser. A 467, 2597 (2011).

[31] D. E. Pelinovsky, Y. S. Kivshar, and V. V. Afanasjev, Phys. Rev. E 54, 2015 (1996).

[32] R. S. Johnson, A Modern Introduction to the Mathematical Theory of Water Waves (Cambridge University Press, Cambridge, UK, 1997).

[33] Solitary Waves in Fluids, edited by R. H. J. Grimshaw (WIT Press, Southampton, 2007).

[34] A. Babanin, Breaking and Dissipation of Ocean Surface Waves (Cambridge University Press, Cambridge, UK, 2011). 